\title{
Identification of anthracene in Comet 1P/Halley
}

\author{
J. Clairemidi ${ }^{1}$, G. Moreels ${ }^{1}$, O. Mousis ${ }^{1}$, and P. Bréchignac ${ }^{2}$ \\ 1 Observatoire de Besançon, Institut UTINAM, CNRS-UMR 6213, BP 1615, 25010 Besançon Cedex, France \\ e-mail: jc@obs-besancon. fr \\ 2 Laboratoire de Photophysique Moléculaire, CNRS, Univ. Paris-Sud, Bât. 210, 91405 Orsay Cedex, France
}

Received 1 February 2008 / Accepted 5 September 2008

\section{ABSTRACT}

\begin{abstract}
Context. Polycyclic aromatic hydrocarbons (PAHs) have been detected in many interstellar medium (ISM) sources. They are considered among the most abundant organic compounds in these environments.

Aims. We aim at identifying the carriers of the near UV fluorescence bands that appear in the spectra of 1P/Halley's inner coma. Methods. Near UV spectra of 1P/Halley were recorded on March 9, 1986 by the three-channel spectrometer onboard the Vega 2 spacecraft at projected distances of between 421 and $932 \mathrm{~km}$. We compare these data to laboratory spectra obtained under laser-induced fluorescence conditions in a jet-cooled molecular beam.

Results. The cometary spectral features are found to be consistent with the laboratory fluorescence spectrum of anthracene. Four main peaks coincide at $363,367.5,373$ and $382.5 \mathrm{~nm}$. We then report the identification of anthracene, a three-ring PAH in the inner coma of $1 \mathrm{P} /$ Halley at projected distances of less than thousand $\mathrm{km}$. We derive an abundance relative to water of $5 \times 10^{-5}$ to $1 \times 10^{-4}$ for this molecule. This new detection adds to the previously reported UV identifications of phenanthrene and pyrene in the innermost coma of $1 / \mathrm{P}$ Halley.

Conclusions. Recently, three small PAHs, namely naphtalene, phenanthrene and pyrene, were identified in dust grains collected by the Stardust probe in the environment of Comet 81P/Wild2. These findings, together with the present identification of anthracene in 1P/Halley's near UV spectra, confirm that similarities exist between the composition of comets and that of the ISM.
\end{abstract}

Key words. space vehicles: instruments - techniques: spectroscopic - comets: general - comets: individual: 1P/Halley line: identification

\section{Introduction}

PAHs have been observed in almost all astrophysical environments, including the diffuse interstellar medium (ISM), the edges of molecular clouds, reflection nebulae, young stellar objects, $\mathrm{H}_{\mathrm{II}}$ regions, star forming regions, planetary nebulae, novae and galaxies (Puget \& Léger 1989; Salama et al. 1996; Verstraete et al. 2001; Vijh et al. 2004, 2005). They are characterized by strong emission bands in the 3-15 $\mu \mathrm{m}$ mid-infrared range (Ehrenfreund \& Charnley 2000; van Dishoeck 2004). Li $\&$ Draine (2001) elaborated a quantitative model for the infrared emission from dust in the diffuse ISM and showed that dust containing carbonaceous grains having properties like PAHs are consistent with observations.

In the Solar system, the three-channel spectrometer (TKS) onboard the Vega2 spacecraft detected unidentified fluorescence bands in the 280-400 $\mathrm{nm}$ range in the innermost coma of comet $1 \mathrm{P} /$ Halley. The cometary spectra were compared to laboratory laser-induced fluorescence spectra of PAHs having 2, 3 or 4 aromatic rings. Two PAHs, namely phenanthrene $\left(\mathrm{C}_{14} \mathrm{H}_{10}\right)$ and pyrene $\left(\mathrm{C}_{16} \mathrm{H}_{10}\right)$, were thus identified by the presence of three peaks in their individual spectra between 342 and $384 \mathrm{~nm}$ (Moreels et al. 1994; Clairemidi et al. 2004). This first identification of PAHs in 1P/Halley was independently confirmed by the detection of an IR emission at $3.28 \mu \mathrm{m}$ by the infrared spectrometer (IKS) onboard the Vega2 probe, due to a mixture of aliphatic species and aromatic polycyclic compounds (Encrenaz et al. 1988).

More recently, naphthalene, phenanthrene and pyrene were identified in comet $81 \mathrm{P} /$ Wild 2 samples collected during the
Stardust mission (Sandford et al. 2006; Clemett et al. 2007). In addition, by using a model for the infrared emission of dust based on a statistical mix of PAHs with an average size $\sim 1 \AA$ for pyrene (Li \& Draine 2001), Lisse et al. (2006) also detected infrared signatures of PAHs at 6.2 and $7.6 \mu \mathrm{m}$ in Spitzer spectra of comet 9P/Tempel 1 ejecta. These new findings led us to re-investigate the UV part of the TKS spectra in order to search for new fluorescence bands of PAHs, as these compounds are quite efficient in emitting near UV and blue luminescence when exposed to UV radiation.

In this paper, we present a set of four spectra of the inner coma of comet 1P/Halley, each one an average of four individual spectra taken at different projected distances between 421 and $932 \mathrm{~km}$ from the nuclei. The data are compared with a fluorescence spectrum of anthracene obtained in the laboratory under simulated cometary conditions. Both types of spectra, cometary and laboratory, present comparable features that allow a confident identification of anthracene in $1 \mathrm{P} /$ Halley.

\section{Spectra of Halley's inner coma}

Spectra of Halley's inner coma in the near UV were obtained on March 9, 1986 with the TKS spectrometer onboard the Vega2 spacecraft. The telescope scanned a rectangular $2^{\circ} \times 1.5^{\circ}$ field of view in the first $150 \mathrm{~min}$ of the observation session and a line with an angular extent of $2^{\circ}$ in the last 30 min preceding the closest approach.

Re-processing of the TKS data was conducted as follows. The dust-scattered continuum due to solar radiation is 


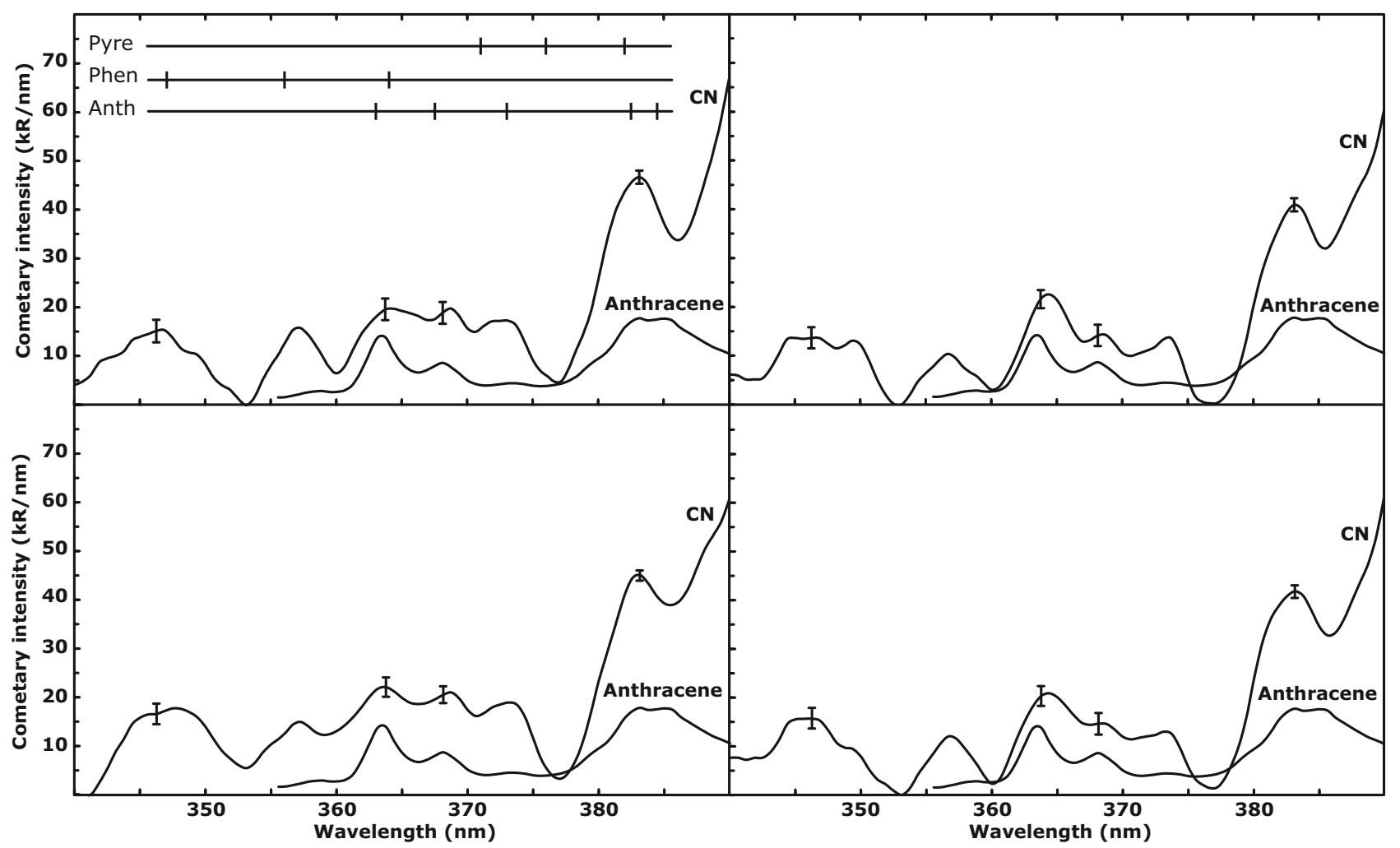

Fig. 1. Comparison of cometary spectra with the laboratory spectrum of anthracene. The $\mathrm{CN}$ emission band is not subtracted from the cometary spectrum. The laboratory spectrum of anthracene, shown with arbitrary intensity units, is presented below the cometary spectrum. The cometary spectrum shown in each panel is an average of four different individual spectra taken at different projected distances $p$ between 421 and $932 \mathrm{~km}$. Four main peaks coincide at 363, 367.5, 373 and $382.5 \mathrm{~nm}$. Error bars are plotted at the wavelengths of several PAH emission bands. Marks in the upper left panel denote the location of the anthracene, phenanthrene and pyrene features.

subtracted. This operation is made by using a reference absolute solar spectrum (Neckel \& Labs 1984; Labs et al. 1987) and applying to it a convolution by a Gaussian function having the same FWHM as the instrument, i.e. $6 \mathrm{~nm}$. The resulting solar intensity $S_{\lambda}$ is then multiplied by an $\alpha$ factor that is determined by adjusting the solar spectrum below the experimental spectrum of intensity $L_{\lambda}$. Both spectra are in contact at one wavelength $\lambda_{0}$ that is measured during the numerical determination of the $\alpha$ coefficient. At the wavelength $\lambda_{0}$, the following relationship is satisfied:

$L_{\lambda 0}-\alpha S_{\lambda 0}=0$.

At other wavelengths, the intensity of molecular emission is given by:

$I_{\lambda}=L_{\lambda}-\alpha S_{\lambda}$.

During this process, the dust-scattered spectrum is assumed to be grey in color, i.e. no color correction is applied to the original data during the subtraction phase. The moderate resolution of the instrument does not allow the resolution of weak and/or closespaced features. It can be noted that the 340-380 nm spectral range between the $\mathrm{NH}$ and $\mathrm{CN}$ bands is practically free of any molecular band in TKS spectra taken at projected distances $p \geq$ $18800 \mathrm{~km}$. However, using high resolution ground-based spectra of comet Austin $(1989 \mathrm{c} 1)$ in the same spectral range, Valk et al. (1992) identified the following weak features: $\mathrm{OH}(0,1)$ at $345 \mathrm{~nm}, \mathrm{CO}_{2}^{+}(\Delta v=0)$ at $352 \mathrm{~nm}, \mathrm{CN}(1,0)$ at $357 \mathrm{~nm}$ and $\mathrm{CO}_{2}^{+}$ $(\Delta v=-1)$ at $368 \mathrm{~nm}$. A detailed long-slit spectrum obtained by Arpigny et al. (1986), with an exposure time of $3 \mathrm{~h} 25 \mathrm{~min}$, also shows weak traces of $\mathrm{CN}(1,0)(357 \mathrm{~nm}), \mathrm{CO}_{2}^{+}(0,0)(352 \mathrm{~nm})$,
$\mathrm{CO}_{2}^{+}(0,1)(368 \mathrm{~nm}), \mathrm{CO}_{2}^{+}(1,2)(369 \mathrm{~nm}), \mathrm{CO}^{+}(4,0)(379 \mathrm{~nm})$ and $\mathrm{OH}^{+}(0,0)(360 \mathrm{~nm})$. However, due to the poor signal to noise ratio, no variation of these features has been detected with $p$, so weak are their intensities.

In the inner coma at $p \leq 3000 \mathrm{~km}$, the situation is quite different. During the approach and encounter period, the Vega2 instrument line of sight continuously scanned an angular field of view of $2^{\circ}$ in order to spatially explore the inner coma. Intense fluorescence broad band emission (10-50 kRayleighs/nm) gradually emerged as the spacecraft approached the comet. The spectra recorded during this session exhibit a noticeable variability which reflects the inhomogeneities of the cometary medium close to the nucleus. A selection of the spectra is displayed in Fig. 1, where a number of fluorescence bands are seen in the 340-390 nm spectral range.

Polyatomic molecules which emit fluorescence radiation in the near UV are numerous. Our search was oriented towards methanol and formaldehyde, since these molecules have been detected in several comets, and towards PAHs which present fluorescence bands in the UV and visible (Jaffé \& Orchin 1962). In the UV, methanol in the gaseous phase shows a weak absorption band at $\lambda \sim 180-200 \mathrm{~nm}$. The molecule is dissociated and produces mainly formaldehyde or, at a lower rate, methoxy radicals (Harrison et al. 1959; Porter \& Noyes 1959; Calvert \& Pitts 1966, p. 444). At shorter wavelengths, $\lambda<150 \mathrm{~nm}$, the dissociation products are methyl and $\mathrm{OH}\left({ }^{2} \Sigma\right)$ radicals (Nee et al. 1985). As a result, methanol does not seem to be a possible fluorescence carrier of the observed emission. In the case of formaldehyde, its fluorescence spectrum presents ten regularly spaced bands at $370,378,387,397,405,414,423,433,443$ and $455 \mathrm{~nm}$ 
(Tang \& Lee 1976; Chatham 1977) but none of them coincide with the reported emission features between 358 and $385 \mathrm{~nm}$.

Among the large family of PAHs, we identified phenanthrene (Moreels et al. 1994) and pyrene (Clairemidi et al. 2004) from TKS spectra of Halley's inner coma. These two PAHs were also identified in the samples of the comet $81 \mathrm{P} /$ Wild 2 collected by Stardust (Sandford et al. 2006; Clemett et al. 2007), and presented as a good match to the material excavated by the Deep Impact experiment from comet 9P/Tempel 1, as observed by the Spitzer Space Telescope at 5-35 $\mu \mathrm{m}$ (Lisse et al. 2006). PAHs are considered to be the best candidates as possible carriers of the cometary emission, because their fluorescence bands are expected to be located in the $330-385 \mathrm{~nm}$ spectral range.

\section{Identification of anthracene}

The identification of some of the bands in comet 1P/Halley as being anthracene has been established on the basis of comparisons between the cometary spectra and emission spectra obtained in the laboratory, under conditions which provide valuable simulation of the astrophysical situation, i.e. super-cooled gas phase molecules in a collision-free regime.

The experimental conditions are described in Hermine (1994), Moreels et al. (1994) and Clairemidi et al. (2004). A heated $\left(50{ }^{\circ} \mathrm{C}\right)$ solid sample reservoir provides a vapour pressure of anthracene which is diluted in a carrier gas $\left(P_{0}=5\right.$ bars of helium) before passing through a heated pulsed nozzle $(0.5 \mathrm{~mm}$ diameter). The anthracene vapour is excited by a pulsed tunable UV beam focused onto the jet at a distance of $\sim 10 \mathrm{~mm}$ from the nozzle. The laser beam is synchronized with the valve operation.

The adiabatic expansion of the gas mixture from the nozzle produces a fast and very efficient cooling of the molecules (kinetic $T<10 \mathrm{~K}$ ). The PAH molecules, travelling with the carrier gas velocity, become rotationally cooled to a temperature close to the kinetic temperature. The molecular density becomes low enough so that the average time between collisions increases faster than the radiative lifetimes of the electronically excited states. These two characteristics of the experiment (very low temperature and absence of collisions) provide an appropriate simulation of the cometary conditions. The fluorescence spectra correspond to the recording of the dispersed emission resulting from excitation at a fixed laser wavelength.

The photophysical properties of the anthracene molecule must be invoked in order to derive from experimental data the expected emission spectrum of the molecule if present in the cometary environment. This is done with the help of the diagrams presented in Fig. 2. The central part of the figure is an energy level scheme partly following the traditional Jablonski's representation. The fluorescence excitation spectrum of jetcooled anthracene is reported on the left hand side of the figure, while the standard solar spectrum is recalled on the right hand side. The central scheme consists of three piles of vibrational energy levels built above the vibrationless electronic singlet states: the ground state $S_{0}$ (right), the first excited singlet $S_{1}$ (middle), and the fourth excited singlet $S_{4}$ (left). Note that the second and third excited singlet states are not represented since, as apparent from the fluorescence excitation spectrum (left), the $\mathrm{S}_{2} \leftarrow \mathrm{S}_{0}$ and $\mathrm{S}_{3} \leftarrow \mathrm{S}_{0}$ transitions have negligible oscillator strengths. The metastable triplet states are not shown either, although they contribute to the reduction of the fluorescence quantum yield by inter-system crossing non radiative transitions. It thus appears that only the vibronic bands belonging to the $S_{1} \leftarrow S_{0}$ and $\mathrm{S}_{4} \leftarrow \mathrm{S}_{0}$ electronic transitions are efficient to excite the fluorescence of anthracene. It is important to note that excitation of the

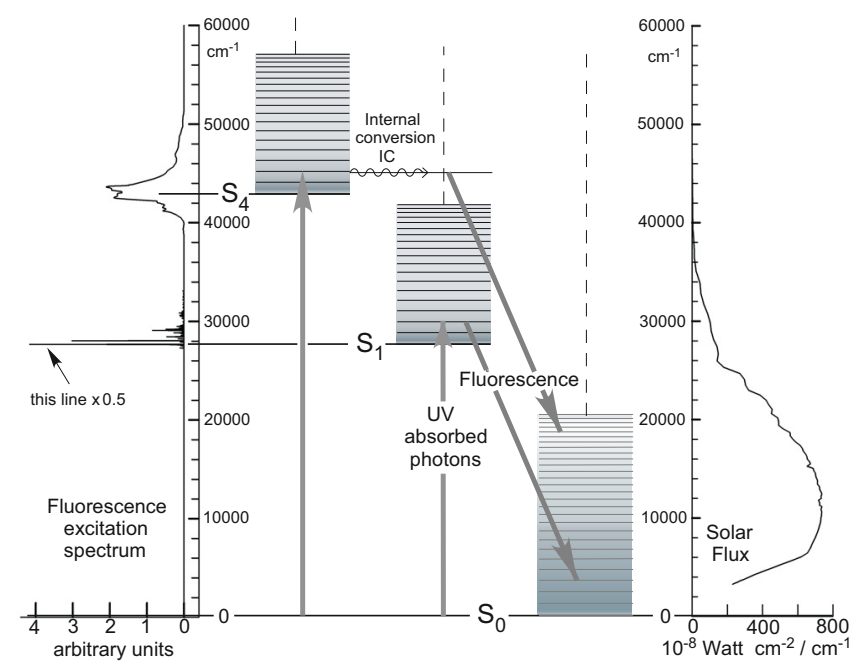

Fig. 2. Energy levels diagram showing the allowed electronic transitions within the singlet states of anthracene. On the left hand side, the fluorescence excitation spectrum of the jet-cooled molecules is reproduced (Hermine 1994). On the right hand side, the solar spectrum is shown for comparison. Note that the $S_{2}$ and $S_{3}$ states are not represented since they do not contribute to the excitation because of their very low oscillator strengths. Triplets that contribute to the reduction of the fluorescence quantum yield by non-radiative transitions are not shown for overall clarity.

$\mathrm{S}_{4}$ state is immediately followed by fast non radiative transitions of internal conversion to the $S_{1}$ state (undulating horizontal arrow in the figure), as shown by the fact that the fluorescence is emitted in the wavelength range of the $S_{1} \leftarrow S_{0}$ transition.

The experimental study shows that excitation through the various vibronic bands of the $S_{1} \leftarrow S_{0}$ transition gives rise to a dispersed emission spectrum which is red-shifted relative to the origin transition at $361 \mathrm{~nm}$, and always similar when the excitation wavelength is varied (Lambert et al. 1984a) and which evolves towards an essentially stable spectrum when intramolecular vibrational redistribution (IVR) is established near $1300 \mathrm{~cm}^{-1}$ of excess energy (Bréchignac \& Hermine 1994; Hermine 1994). Such an evolution towards a stable spectrum is also observed when excitation is achieved at different wavelengths through the broad features assigned to the $S_{4} \leftarrow S_{0}$ transition, but the corresponding dispersed emission spectrum is quite different and featureless (Bréchignac \& Hermine 1994). The contribution of each of these two average emission spectra to the expected cometary spectrum results from taking into account the solar photons flux in the two spectral windows for excitation, as well as the adequate fluorescence efficiency in each case. It is clear from the solar spectrum in Fig. 2 that the flux available for excitation to $\mathrm{S}_{4}$ is significantly smaller than it is for excitation to $S_{1}$. The quantum yield is also reduced from 0.67 (at the origin of $S_{1}$ ) to 0.02 (at the high energy side of the $S_{4}$ band). Thus, the contribution of the fluorescence from $S_{1}$ is expected to be about 20 times larger than that from $\mathrm{S}_{4}$, which consequently can be neglected. The laboratory photophysical studies thus led us to adopt the spectrum with $\lambda_{\mathrm{exc}}=344 \mathrm{~nm}$ reported by Lambert et al. (1984b), which is reproduced in the four panels of Fig. 1.

We selected for this figure cometary spectra where the fluorescence bands of anthracene at 363, 367.5, 373, 382.5 and $384.5 \mathrm{~nm}$ are present. As the medium probably contains a mixture of PAHs, the simultaneous presence in the four spectra of the fluorescence bands of anthracene at 363, 367.5, 373, 
382.5-384.5 $\mathrm{nm}$ constitutes a strong criterion for identifying this molecule. The main fluorescence bands of phenanthrene located at $347,356,364 \mathrm{~nm}$ are also seen. In the case of pyrene, the bands located at 371, 376 and $382 \mathrm{~nm}$ are barely seen in the spectra that were chosen in the present work. As a result, the spectra shown in Fig. 1 suggest the identification of anthracene in Halley's comet but also confirm the presence of phenanthrene.

Following the same approach as in our previous cometary PAH work (Moreels et al. 1994; Bréchignac \& Hermine 1994, Clairemidi et al. 2004), i.e. relying on the absolute oscillator strength for $\mathrm{S}_{1} \leftarrow \mathrm{S}_{0}$ absorption and fluorescence quantum yields from $\mathrm{S}_{1}$ (near $\lambda_{\mathrm{exc}}=344 \mathrm{~nm}, Q \simeq 0.2$ ) (Hermine 1994), integrating the cometary intensity in anthracene bands (from Fig. 1) and assuming the expansion velocity is $1 \mathrm{~km} \mathrm{~s}^{-1}$, it is possible to derive the production rate of anthracene: $Q_{\text {anthracene }} \sim$ $10^{26}$ molecules s ${ }^{-1}$.

This value may be compared with the production rate of $\mathrm{C}_{2}$, estimated to be $6 \times 10^{27}$ molecules $\mathrm{s}^{-1}$ for March 9, the encounter day (Krasnopolsky et al. 1986). Comparing the production rate of anthracene to the estimated production rate of water mentioned by Krasnopolsky et al. (1986), $Q_{\mathrm{H}_{2} \mathrm{O}}=2 \times$ $10^{30}$ molecules s ${ }^{-1}$, and by Encrenaz et al. (1988) for P/Halley, $Q_{\mathrm{H}_{2} \mathrm{O}}=10^{30}$ molecules $\mathrm{s}^{-1}$, we derive a maximum abundance of anthracene relative to water of $5 \times 10^{-5}$ to $1 \times 10^{-4}$. Note that Bockelée-Morvan et al. (1995) found a lower PAH abundance relative to water $\left(\sim 1.5 \times 10^{-6}\right.$ to $\left.10^{-5}\right)$ in a set of seven comets, but their derived abundances depend on modeling assumptions which are still uncertain and there is a significant diversity among comets, namely in the dust/gas ratio and in the composition of the coma.

In the same conditions the cometary intensity in phenanthrene bands from Fig. 1 would lead to $Q_{\text {phenanthrene }}$ ranging from $6 \times 10^{26}$ to $10^{27}$ molecules/s. The order of magnitude is similar to our previous determination (Moreels et al. 1994) although weaker by a factor of $\simeq 2$. Such a difference can be explained by the fact that the spectra in Fig. 1 have been selected to clearly show the anthracene bands, in contrast to our previous work. The higher abundance of phenanthrene compared to anthracene could be explained by the fact that it is more stable than anthracene, as shown by the comparison of the resonance energy of their polycyclic structures (see e.g. Fig. 2 p. 4, Bjørseth 1983).

This reveals a clear variability in the spectra which must have its origin in the heterogeneity of the material composition along the time-varying line-of-sight. Such a behavior is fully consistent with the evidence of highly anisotropic jets in Halley's coma, and is expected to be emphasized if the PAH molecules are indeed outgassed from the dust particles themselves, possibly released by the fragmentation of clumps. Such a process is also proposed by Green et al. (2007) to explain the impact count data recorded from the Wild 2 coma during the Stardust closest approach. The measured fluxes are attributed to bursts and swarms that reveal a highly non-uniform distribution for the Wild 2 coma material.

\section{Spatial distribution of the emission}

A relatively detailed description of the region close to the nucleus can be retrieved from the thousands of spectra of the explored inner coma transmitted by the three-channel spectrometer. Once the geometrical parameters are calculated, radial profiles can be drawn for any wavelength of the instrument spectral range.

Radial profiles of the individual band intensities can be plotted as a function of the projected distance $p$. Two radial profiles

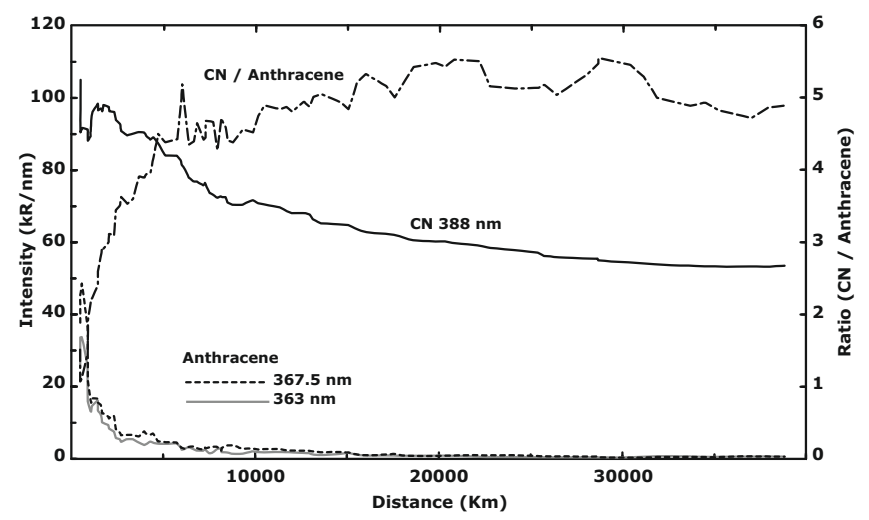

Fig. 3. Intensity radial distribution as a function of $p$, projected distance between the nucleus and the line of sight. Left-hand scale: intensities in $\mathrm{kR} / \mathrm{nm}$ of the broad-band emission peaks of anthracene at 363 and $367.5 \mathrm{~nm}$. The intensity of the CN band at $388 \mathrm{~nm}$ is increased by an offset of $20 \mathrm{kR}$ for the clarity of the curves. Right-hand scale: intensity ratio ( $\mathrm{CN}$ at $388 \mathrm{~nm} /$ average of the 2 peaks at 363 and $367.5 \mathrm{~nm})$. The ratio increases from 0.87 to 6 with $p$ increasing from 400 to $40000 \mathrm{~km}$.

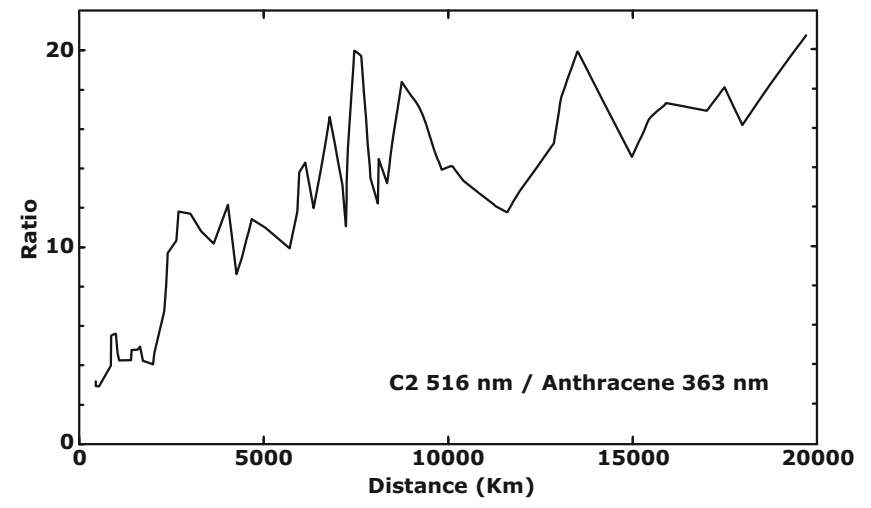

Fig. 4. Intensity ratio $\left(C_{2}\right.$ at $516 \mathrm{~nm} / \mathrm{emission}$ peak at $\left.363 \mathrm{~nm}\right)$. The ratio increases from 3 to 20 with $p$ increasing from 400 to $20000 \mathrm{~km}$.

of anthracene at wavelengths 363 and $367.5 \mathrm{~nm}$ are presented in Fig. 3. For comparison, the intensity of the $\mathrm{CN}$ band at $388 \mathrm{~nm}$ is also plotted. A fourth curve shows the variation with $p$ of the intensity ratio $\mathrm{CN}$ at $388 \mathrm{~nm} / \mathrm{emission}$ peak at $363 \mathrm{~nm}$ and $367.5 \mathrm{~nm}$. Anthracene exhibits a typical parent molecule profile, with a maximum at the nucleus and a sharp decrease as a function of the projected distance. Figure 4 presents the variation with $p$ of the intensity ratio $\mathrm{C}_{2}$ at $516 \mathrm{~nm} / \mathrm{emission}$ peak of anthracene at $363 \mathrm{~nm}$. The ratio rises to a value $\sim 20$, which indeed corresponds to the sensivity limit. $\mathrm{CN}$ and $\mathrm{C}_{2}$ have daughter molecule profiles with very long scalelengths.

Several parent molecules $\mathrm{P}(\mathrm{CN})$ have been proposed for $\mathrm{CN}$ : $\mathrm{HCN}, \mathrm{CH}_{3} \mathrm{CN}, \mathrm{HC}_{3} \mathrm{~N}$ and $\mathrm{C}_{2} \mathrm{~N}_{2}$ (Bockelée-Morvan \& Crovisier 2002; Fray et al. 2005). The shape of the $I(p)$ curve for the newly detected emission is, to a first approximation, of the $1 / p$ type. Such a $1 / p$ variation is a strong argument to prove that the identified PAH molecule is either a parent molecule or is released inside the coma by dust particles at low velocities.

The present data, combined with those derived from other sets of spectra previously reported by Moreels et al. (1994) and Clairemidi et al. (2004), allow a comparison of the spatial distribution of the three detected PAHs to be made. Figure 5 shows the variation with $p$ of the intensity of pyrene, anthracene and phenanthrene measured by TKS. Using a simple uniform expansion model, a value of the scale length for the detected 


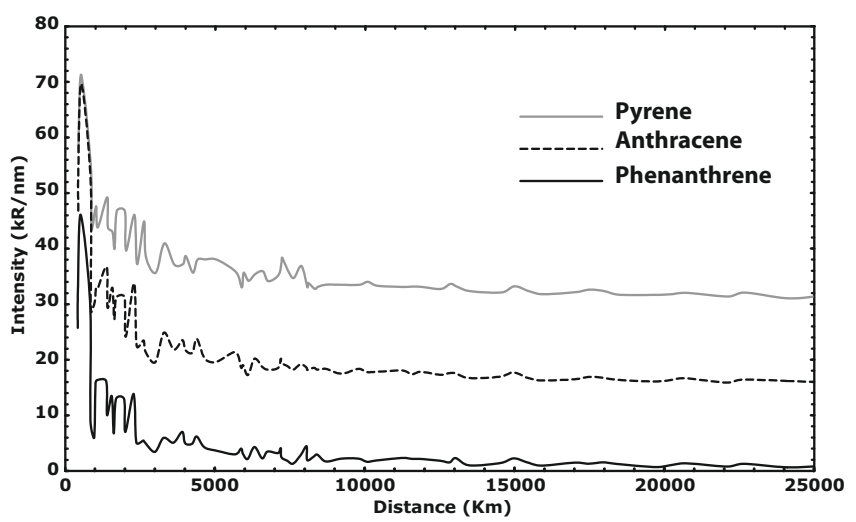

Fig. 5. Intensities of the three PAHs measured by TKS as a function of $p$, projected distance between the nucleus and the line of sight. For clarity, the upper curve for pyrene is shifted upward by $30 \mathrm{kR} / \mathrm{nm}$ and the middle curve for anthracene by $15 \mathrm{kR} / \mathrm{nm}$. The lower curve is for phenanthrene.

PAH can be calculated by integrating the following density equation:

$n\left(r_{\mathrm{n}}\right)=g \frac{Q \exp \left(-r_{\mathrm{n}} / v \tau_{0}\right)}{4 \pi v r_{\mathrm{n}}^{2}}$,

where $g$ is the fluorescence efficiency, $Q$ the production rate, $r_{\mathrm{n}}$ the radial distance to the cometary nucleus, $v$ the expansion velocity and $\tau_{0}$ the considered $\mathrm{PAH}$ lifetime. Assuming that $v=1 \mathrm{~km} \mathrm{~s}^{-1}$, we find a value of $\tau_{0} \sim(1.2 \pm 0.2) \times 10^{5} \mathrm{~km}$ for anthracene. Such a high value for $\tau_{0}$ suggests that PAHs are also produced locally by dust grains in the coma. Indeed, as the band intensities are not spatially homogeneous, the PAHs are probably released by disintegration of dust particle clumps, in a process similar to that found by Stardust in the Wild 2 coma (Green et al. 2007). In addition, the camera onboard Giotto also showed evidence of highly anisotropic emission from Halley's nucleus, mainly sunward, and dominated by pronounced jets of material (Keller et al. 1987). Such jets of CN and NH were also detected by TKS (Clairemidi et al. 1990).

$\mathrm{C}_{2} \mathrm{~N}_{2}$ and $\mathrm{HC}_{3} \mathrm{~N}$ appear as possible candidates, but the first one is very difficult to detect and the second not abundant enough to explain the $\mathrm{CN}$ quantity in the coma. In the case of $\mathrm{C}_{2}$, it is produced at a high excitation temperature in the jets or in diffuse sources, and lower temperature in the surrounding coma (Laffont et al. 1998). Determining the nature of its parent molecule(s) $\mathrm{P}\left(\mathrm{C}_{2}\right)$ is still an open question. Photodestruction of PAHs at Ly $\alpha$ might be a path for releasing $\mathrm{C}_{2} \mathrm{H}_{2}$ breaking into $\mathrm{C}_{2} \mathrm{H}, \mathrm{CH}$ and $\mathrm{C}_{2}$ (Calvert \& Pitts 1966; Okabe 1978). The production of vibronically excited $\mathrm{C}_{2}\left(\mathrm{c}^{3} \Sigma_{u}^{+}, \mathrm{d}^{3} \Pi_{g}\right)$ has been observed in the laboratory by Moutou et al. (1997) under excimer laser excitation of another PAH, perylene. Some of the concerned bands only very recently have been assigned thanks to the work of Kokkin et al. (2006). Further studies of thermal or photodestruction of carbonaceous compounds might provide pertinent answers to the problem of $\mathrm{P}(\mathrm{CN})$ and $\mathrm{P}\left(\mathrm{C}_{2}\right)$ (Ehrenfreund et al. 2002).

\section{Summary and discussion}

The recent identification of PAHs in the cometary dust grains collected during the Stardust mission (Clemett et al. 2007) led us to re-investigate the spectra of Halley's inner coma recorded by the TKS spectrometer. We compared the cometary data with laser induced fluorescence spectra obtained under cooled molecular jet conditions. Using anthracene vapour, four main peaks located at $363,367.5,373$ and $382.5 \mathrm{~nm}$ were identified in the laboratory spectrum of the 3-ring PAH, as well as in the cometary spectra. In addition, the spatial distribution of the emission exhibits a $p^{-1}$ variation which shows that its carrier, anthracene, is emitted by the nucleus or by dust packs in the coma.

The identification of anthracene confirms the previously reported detection of phenanthrene (Moreels et al. 1994), and pyrene (Clairemidi et al. 2004). It should be noted that the structure of individual spectra is not uniform for the whole set of TKS data. This reveals some heterogeneity in the composition of clumps or of dust grains as pointed out by the Stardust results (Clemett et al. 2007).

The chemistry capable of producing such PAHs is likely to create, under similar conditions, a whole family of polycyclic aromatic molecules. In particular, the presence of naphthalene is not excluded from our spectra but it is difficult to determine definitely because the expected spectrum presents only one broad $(\sim 25 \mathrm{~nm})$ band without characteristic features (Bréchignac \& Hermine 1994). These detections imply that other PAHs might also be present in comet Halley and give rise to characteristic emission spectra. Such spectra would occur at different wavelengths as shown by separate studies concerning molecules of similar sizes in the laboratory, and need to be further investigated. These three identifications have been made possible by the absence of any other molecular emission in the whole spectral range covered where anthracene, pyrene and phenanthrene fluorescence occurs ( from 340 to $390 \mathrm{~nm}$ ).

The identifications provide a full complement to the detection of an IR feature at $3.28 \mu \mathrm{m}$ and its assignment to an $\mathrm{X}-\mathrm{CH}$ organic compound. Lisse et al. (2007) reported the detection of the IR signature of PAHs in comets Tempel1, Hale-Bopp and in the Young Stellar Object (YSO) HD 100546. This is consistent with the detection of strong PAH emission in Herbig Ae/Be stars (Sloan et al. 2005). The material of the disk surrounding these stars is very similar to that found in comets (e.g. HD 100546 vs. Comet Hale-Bopp) and seems most likely in the regime of building giant planets out of cometary planetesimals (van den Acker et al. 1997; Waelkens et al. 1997; Bouwman et al. 2003; Lisse et al. 2007).

Measuring the fluorescence radiation of small PAHs in the near-UV is an effective way to identify specific species. Recently, Vijh et al. (2004, 2005) observed a blue luminosity in the Red Rectangle nebula. They compared their spectra with dispersed fluorescence laboratory spectra of anthracene and pyrene (Bréchignac \& Hermine 1994). They proposed anthracene as a likely carrier of the observed blue luminosity. The Red Rectangle is a proto-planetary nebula with a central star, HD 44179, showing a large mass loss. Its IR spectrum exhibits the five "unidentified" IR emission bands at 3.3, 6.2 , 7.8, 8.6 and $11 \mu \mathrm{m}$ identified as being of "highly characteristic of polycyclic aromatic compounds" by Léger \& Puget (1984). Many efforts have been made to define criteria for identifying PAHs through their phosphorescence or far-IR emission (Joblin et al. 2002; Mulas et al. 2006). The identification of small neutral polycyclic molecules in Halley's inner coma and in the Red Rectangle nebula emphasizes the similarity of composition that exists between minor bodies formed in the outer primitive nebula and the interstellar medium. These new data support the idea that comets can help trace the interstellar medium input into the primitive solar nebula, even if this matter might have been strongly altered or even completely re-processed, down to the molecular level, by strong mixing within the disk, as the new Stardust and Deep Impact results suggest. 
Acknowledgements. Most grateful thanks are due to Marie-Jeanne for invaluable help during the TKS-Vega program. Sébastien Lebonnois and an anonymous referee are acknowledged for their fruitful comments.

\section{References}

Arpigny, C., Dossin, F., Manfroid, J., Danks, A. C., \& Lambert, D. L. 1986, Msngr, 45, 8

Bjørseth, A. 1983, Handbook of polycyclic aromatic hydrocarbons (New York: Marcel Dekker, Inc.), 10016

Bockelée-Morvan, D., \& Crovisier, J. 2002, EM\&P, 89, 53

Bockelée-Morvan, D., Brooke, T. Y., \& Crovisier, J. 1995, Icarus, 116, 18

Bouwman, J., de Koter, A., Dominik, C., \& Waters, L. B. F. M. 2003, A\&A, 401, 577

Bréchignac, P., \& Hermine, P. 1994, in Molecules and Grains in Space, ed. I. Nenner (New York: AIP) AIP Conf. Proc., 312, 613

Calvert, J. G., \& Pitts, J. N. Jr. 1966, Photochemistry (New York: Wiley)

Chatham, R. E. 1977, New Investigation of Nitrogen Laser Induced Fluorescence in Formaldehyde: Spectra and Spectrally Resolved Lifetime, Ph. Dissertation, State University of New York at Stony Brook, No. 256944

Clairemidi, J., Moreels, G., \& Krasnopolsky, V. A. 1990, Icarus, 86, 115

Clairemidi, J., Bréchignac, P., Moreels, G., \& Pautet, D. 2004, P\&SS, 52, 761

Clemett, S. J., Nakamura-Messenger, K., McKay, D. S., \& Sandford, S. A. 2007, LPI, 38, 2091

Ehrenfreund, P., \& Charnley, S. B. 2000, ARA\&A, 38, 427

Ehrenfreund, P., Rodgers, S. D., \& Charnley, S. B. 2002, EM\&P, 89, 221

Encrenaz, T., D'Hendecourt, L., \& Puget, J. L. 1988, A\&A, 207, 162

Fray, N., Bénilan, Y., Cottin, H., Gazeau, M.-C., \& Crovisier, J. 2005, P\&SS, 53, 1243

Green, S. F., McBride, N., Colwell, M. T. S. H., et al. 2007, ESASP, 643, 35

Harrison, A. J., Cederholm, B. J., \& Terwilliger, M. A. 1959, J. Ch. Ph., 30, 355

Hermine, P. 1994, Ph.D.T., Université Paris XI, Orsay

Jaffé, H. H., \& Orchin, M. 1962 Theory and application of ultraviolet spectroscopy, (New York: Wiley)

Joblin, C., Toublanc, D., Boissel, P., \& Tielens, A. G. G. M. 2002, MolPh, 100, 3595

Keller, H. U., Arpigny, C., Barbieri, C., et al. 1986, Nature, 321, 320
Kokkin, D. L., Reilly, N. J., Morris, C. W., et al. 2006, J. Chem. Phys., 125, 231101

Krasnopolsky, V. A., Gogoshev, M., Moreels, G., et al. 1986, Nature, 321, 269

Labs, D., Neckel, H., Simon, P. C., \& Thuillier, G. 1987, SoPh, 107, 203

Laffont, C., Rousselot, P., Clairemidi, J., \& Moreels, G. 1998, P\&SS, 46, 585

Lambert, W. R., Felker, P. M., Syage, J. A., \& Zewail, A. H. 1984a, J. Chem. Phys., 81, 2195

Lambert, W.R, Felker, P. M., \& Zewail, A. H. 1984b, J. Chem. Phys., 81, 2217

Léger, A., \& Puget, J. L. 1984, A\&A, 137, L5

Li, A., \& Draine, B. T. 2001, ApJ, 554, 778

Lisse, C. M., Van Cleve, J., Adams, A. C., et al. 2006, Science, 313, 635

Lisse, C. M., Kraemer, K. E., Nuth, J. A., Li, A., \& Joswiak, D. 2007, Icarus, 187,69

Moreels, G., Clairemidi, J., Hermine, P., Bréchignac, P., \& Rousselot, P. 1994, A\&A, 282, 643

Moutou, C., Verstraete, L., Bréchignac, P., Piccirillo, S., \& Léger, A. 1997, A\&A, 319,331

Mulas, G., Malloci, G., Joblin, C., \& Toublanc, D. 2006, A\&A, 456, 161

Neckel, H., \& Labs, D. 1984, SoPh, 90, 205

Nee, J. B., Suto, M., \& Lee, L. C. 1985, Chem. Phys., 98, 147

Okabe, H. 1978, Photochemistry of small molecules (New York: Wiley)

Porter, R. P., \& Noyes, A. 1959, J. Am. Chem. Soc., 81, 2307

Puget, J. L., \& Léger, A. 1989, ARA\&A, 27, 161

Salama, F., Bakes, E. L. O., Allamandola, L. J., \& Tielens, A. G. G. M. 1996, ApJ, 458, 621

Sandford, S. A. Aléon, J., Alexander, C. M. O. D', et al. 2006, Science, 314, 1720

Sloan, G. C., Keller, L. D., Forrest, W. J., et al. 2005, ApJ, 632, 956

Tang, K. Y., \& Lee, E. K. C. 1976, Chem. Phys. Lett., 43, 2

Valk, J. H., O'dell, C. R., Cochran, A. L., et al. 1992, ApJ, 388, 621

van den Acker, M. E., The, P. S., Tjin A Djie, H. R. E., et al. 1997, A\&A, 324, L33

van Dishoeck, E. F. 2004, ARA\&A, 42, 119

Verstraete, L., Pech, C., Moutou, C., et al. 2001, A\&A, 372, 981

Vijh, U. P., Witt, A. N., \& Gordon, K. D. 2004, ApJ, 606, L65

Vijh, U. P., Witt, A. N., \& Gordon, K. D. 2005, ApJ, 619, 368

Waelkens, C., Malfait, K., \& Waters, L. B. F. M. 1997, EM\&P, 79, 265 